\title{
NEUROPHYSIOLOGICAL AND BEHAVIOURAL PERTURBATIONS IN Caenorhabditis elegans EXPOSED TO ORGANOPHOSPHATE PESTICIDES
}

\author{
Rajul Jain (D), Priyanka Gautam*
}

Bioinformatics Laboratory, Department of Zoology, Dayalbagh Educational Institute (Deemed University), Dayalbagh Agra-282005, India.

Received - April 20, 2021; Revision - June 01, 2021; Accepted - June 10, 2021

Available Online - June 25, 2021

DOI: http://dx.doi.org/10.18006/2021.9(3).343.352

\section{KEYWORDS \\ Organophosphate \\ Behavioural toxicity \\ Developmental toxicity \\ Neurodegeneration \\ Caenorhabditis elegans}

\begin{abstract}
The ubiquitous use of pesticides all over the world leads to adverse effects on both targets as well as non-target species. The extensive and uncontrolled use of organophosphates (OPs), a large group of pesticidal compounds in agricultural and household products are resulting in high exposure to humans. This research has been carried out to study the adverse effect of OPs i.e., chlorpyrifos, trichlorfon, and disulfoton on model organism Caenorhabditis elegans to evaluate their behavioural as well as developmental toxicity at different time intervals i.e., 4, 24, 48, and 72 hours (hrs) of exposure. A significant difference was observed in all the behavioural endpoints like locomotion, egg-laying, offspring count, and learning along with developmental parameters like mortality, paralysis, and growth rendering from moderate to high toxic effects. Based on the above screening, trichlorfon resulted in glutamatergic and cholinergic neurodegeneration along with elevated autofluorescence. Loss in Yellow fluorescent Protein (YFP) and Green Fluorescent Protein (GFP) was recorded by $57.96 \%$ and $30.52 \%$ using transgenic strains OH11124 (otIs388 [eat-4(fosmid)::SL2::YFP::H2B + (pBX)pha-1(+)] III) and OH13083 (otIs576 [unc-17(fosmid)::GFP + lin-44::YFP]). These results have shown the biological potency of toxicants in $C$. elegans and pave the way forward to provide insight into various neurogenerative diseases in humans.
\end{abstract}

* Corresponding author

E-mail: drpriya18@gmail.com (Priyanka Gautam)

Peer review under responsibility of Journal of Experimental Biology and Agricultural Sciences.

Production and Hosting by Horizon Publisher India [HPI] (http://www.horizonpublisherindia.in/).

All rights reserved.
All the articles published by Journal of Experimental Biology and Agricultural Sciences are licensed under a Creative Commons Attribution-NonCommercial 4.0 International License Based on a work at www.jebas.org.

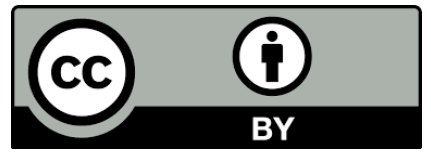




\section{Introduction}

Organophosphates (OPs) represent a common class of pesticides that are extensively used not only in agricultural operations but also commercially and residentially affecting a large number of people directly or indirectly (Muñoz-Quezada et al., 2016; Reynoso et al., 2019). OPs act as acetylcholine-esterase (AChE) inhibitors causing both short-term and long-term behavioural deficits (Salim \& Rajini, 2014; John et al., 2021). Due to the target specificity of these OPs, these pesticides are known to cause neurodegenerative diseases affecting people at any age but showing their prominent effect only at later stages of life (Hargreaves, 2012; Sánchez-Santed et al., 2016; Mukherjee \& Gupta, 2020). However, mechanisms resulting in persistent effects and neurodegeneration remain controversial and difficult to detect (Lewis et al., 2013).

Earlier, synthetic organophosphate compounds were developed as nerve gas agents during the 1940s, but in later years, OPs were approved as insecticides with controlled concentrations (Mukherjee \& Gupta, 2020; John et al., 2021). Preliminary studies have proven a robust link between neurodegenerative diseases like Alzheimer's and Parkinson's to exposure of pesticides, yet the association between specific pesticides and these diseases have not been well studied (Caldwell et al., 2020). Malfunctioning of the cholinergic cells seated in the basal forebrain has been shown to associate Alzheimer's disease (AD) with defective memory and cognition (Muir, 1997; Roldan-Tapia et al., 2006; Meyer-Baron et al., 2015). The propensity of OPs to induce $\mathrm{AD}$ via varied mechanisms have been hypothesized as an elevated beta-amyloid precursor protein, hyperphosphorylated tau protein, oxidative stress, apoptosis, disrupted neurogenesis, and many more (Terry, 2012; Ruszkiewicz et al., 2018). Along with cholinergic, the glutamatergic hypothesis has also been extensively studied in association with Alzheimer's disease. These findings largely account for the neuropsychological disruption and pave the way to the ongoing advancement in the pathology of AD patients (Francis et al., 1999; Caldwell et al., 2020).

The majority of the studies illustrate the effects of the higher concentration of toxicants as monitoring the lower concentrations of pesticides needs more sensitive and reliable methods. Advanced analytical techniques, such as High-Throughput Screening (HTS), LCMS (Liquid Chromatography coupled to Mass Spectrometry), high-performance liquid chromatography (HPLC), etc. are currently used in monitoring routes of pesticide penetrance (Jain et al., 2012). Although these methods employ notable understanding but then the preceding disadvantages include sophisticated instrumentation, exhausting inputs like time and money, and unavailability of equipment. Thus, these applications bear drawbacks in correlating low or permissible pesticide concentration with environmental toxicity and its consecutive health. Therefore, some quick, sensitive, and reliable monitoring methods are essential for time-to-time monitoring of low-dosage pesticide residues in the practical approaches. Hence, biological rather than analytical monitoring methods may be a reasonable alternative in screening the variable range of pesticides regularly (Meyer \& Williams, 2014).

The nematode $C$. elegans is an exceptionally well-studied model organism. It is highly explicable due to its small size $(\sim 1 \mathrm{~mm})$, short life cycle (3-4 weeks), transparent body, ease of maintenance, and its fully sequenced genome making it apt for further investigations related to genotoxicity, xenobiotics, germline toxicity and neurodegeneration where they have found its accountable relevance to humans (Harlow et al., 2016; Queirós et al., 2019).

As model organisms have constantly played a vital role in understanding various disease and their complex pathways, this study is an attempt to provide a convenient way for researchers to understand the neurophysiological and behavioural perturbations caused by organophosphates exposure through various experiments performed on C. elegans (a neural model for humans).

\section{Materials and Methods}

\subsection{Materials}

The model organism used in the study was C. elegans N2 (wildtype strain), which was fed upon bacterial food strain i.e., Escherichia coli OP50. Both were received from the Caenorhabditis Genetics Center (CGC, Minneapolis, MN). For neurodegeneration, OH11124 (otIs388 [eat4(fosmid)::SL2::YFP::H2B + (pBX) pha-1(+)] III ) expressing glutamatergic neuron with YFP expression and OH13083 (otIs576 [unc-17(fosmid)::GFP + lin-44::YFP]) expressing cholinergic neuron with GFP expression were purchased from CGC. Pesticides used in the study were Chlorpyrifos, Disulfoton and Trichlorfon purchased from Sigma Aldrich. All other chemicals used in the experiments were of analytical grade.

\subsection{Worm maintenance and Treatment}

C. elegans were cultured at $20^{\circ} \mathrm{C}$ on NGM plates preseeded with the lawn of Escherichia coli strain OP50 following the methodology of Stiernagle (Stiernagle, 2006). Hypochlorite treatment was used to obtain age-synchronous populations of N2 worms (Rajini et al., 2008; Bradford et al., 2020).

The concentration of the toxicants i.e., Chlorpyrifos (0.5\%), Disulfoton (1\%), and Trichlorfon (2\%) were decided according to their percent used in the commercially available products against pest. Acetone, as a solvent was used for the preparation of control plates, and subsequently pesticides were dissolved in acetone to achieve the final concentration in NGM before poring to the plate for all the toxicants in triplicates. 


\subsection{Behavioural and Developmental Endpoints}

The propensity of these toxicants was analyzed by comparing the behavioural and developmental endpoints between treated and untreated worms. For every parameter, treatment was given to $10 \pm$ 1 worms and along with their respective control in triplicate. Readings for different parameters were taken at 4, 24, 48, and 72 hrs of exposure.

\subsubsection{Locomotion}

Gamuts of chemicals and environmental stresses have been linked to deteriorating the neural network of nematodes causing faulty locomotion. To validate OP toxicants the downstream, $10 \pm 1$ adult worm were examined by tracking their locomotion using a $10 \mathrm{X}$ stereo-zoom microscope. Deciphering locomotion, paralysis formed the foremost visible parameter of toxicity. The worm which showed only a slight movement of the head after teasing with platinum wire were scored paralyzed (Tejeda et al., 2016).

\subsubsection{Egg Laying}

The number of eggs laid was determined by exposing $10 \pm 1$ agesynchronized adult worms with all the toxicants in triplicates alone with their control under a 10X stereo-zoom microscope at 4, 24, 48, and $72 \mathrm{hrs}$ of exposure (Bradford et al., 2020).

\subsubsection{Offspring Count}

Age synchronized $10 \pm 1$ adult worms (L4 stage) were exposed to all the toxicants up to $72 \mathrm{hrs}$. The effects of toxicants on the development of nematodes were investigated by counting the number of offspring of all the stages at 4, 24, 48, and $72 \mathrm{hrs}$ in all the test plates along with a control group (Roh \& Choi, 2011; Bradford et al., 2020).

\subsubsection{Learning}

C. elegans tends to move towards its food even in tricky situations. Any change in its learning behaviour corresponds to an alteration in its nervous system. In this parameter, after 72 hours of exposure, a viable worm from treated as well as control plates were picked up and transferred to another test plate. The test plate contained an equidistant well of food for $E$. coli i.e., OP50, and solvent which was used for the final preparation of pesticidal concentration. The worm was placed at the center of the plate and allowed to follow its instincts (Tejeda et al., 2016).

\subsubsection{Mortality}

This assay was performed to determine the mortality rate derived after toxicity. For this, $10 \pm 1$ young adult were transferred to NGM plates in the presence of food at $20^{\circ} \mathrm{C}$. Then, the number of dead worms was counted through visual inspection using a stereo-zoom microscope at 4, 24, 48, and 72 hrs of exposure (Bradford et al., 2020).

\subsubsection{Growth}

The toxic effect on growth was check by treating the L1 stage of worms with the toxicants for $72 \mathrm{hrs}$ of exposure. The growth of $C$. elegans was confirmed by measuring the body length before and after exposure to the pesticides with a $10 \mathrm{X}$ microscope using a scaled lens (Tejeda et al., 2016; Bradford et al., 2020).

\subsubsection{Neurodegeneration and Autofluorescence}

Transgenic strains OH11124 (otIs388 [eat4(fosmid)::SL2::YFP::H2B + (pBX) pha-1(+)] III) and OH13083 (otIs576 [unc-17(fosmid)::GFP + lin-44::YFP]) were used to study degeneration of cholinergic and glutamatergic neuron respectively. Worms were exposed to the above-tested concentration of trichlorfon and analyzed under a fluorescence microscope to visualize the GFP tagged neurons after pesticide exposure (Bradford et al., 2020).

Autofluorescence in C. elegans is a natural phenomenon seen in the intestine and uterus as the worm ages (Pincus et al., 2016). Mitochondria, intracellular lysosome-derived granules, and extracellular collagen mark the key sources of autofluorescence (Monici, 2005).

\subsubsection{Statistical analysis}

Each assay was conducted in triplicate on three different days with their respective controls. Data were expressed as mean \pm SEM. Students t-test was conducted at the $\mathrm{p}=0.05$ level of significance.

\section{Results}

An organism's overall behaviour and development is the accumulative cooperation of varied intrinsic as well as extrinsic factors where the assessment of certain physiological changes can serve as a responsive indicator of prevailing consequences. One of the major aspects of performing behavioural and physiological studies is the establishment of a specific endpoint (Cole et al., 2004). The utility of $C$. elegans was assessed as a model organism to screen pesticides.

\subsection{Locomotion}

The period of exposure to a response relationship was seen for all three tested pesticides. The movement of worms was not decreased when exposed to disulfoton, but a clear period of exposure to response relationship was exhibited in trichlorfon and chlorpyrifos. The movement of the worm was reduced where the response leveled off as the time of exposure increased. There was 100\% decrease in the locomotion of chlorpyrifos while trichlorfon had 
$50 \%$ reduction in the movement of worms. Percent paralysis was quantified by counting the number of worms paralyzed at every exposure hour. Chlorpyrifos and trichlorfon showed overall paralysis by $60 \%$ and $30 \%$ while disulfoton showed paralysis by $30 \%$ as shown in figure $1(\mathrm{~A})$.

\subsection{Egg-laying}

Worms exposed to OPs exhibit a significant reduction in the egglaying since early 4 hrs (Figure1B). It is known that the egg-laying capacity of the worm is maximum at $24 \mathrm{hrs}$, which gradually decreases thereby. After $24 \mathrm{hrs}$ of OPs exposure, a decrease of 87 , 67 , and $80 \%$ was being noticed in egg count than the control for chlorpyrifos, disulfoton, and trichlorfon respectively. While at 48 hrs, this decrease in egg-laying was $100 \%$ in the case of chlorpyrifos \& trichlorfon with $62 \%$ for disulfoton. There was no egg-laying seen at $72 \mathrm{hrs}$ of pesticide exposure in all three OPs.

\subsection{Offspring count}

Similarly, the offspring count was evaluated in all three OPs. Initially, there were no offspring seen in all plates at 4 hrs of exposure. As seen from figure1(C), the highest effect was seen in chlorpyrifos where it showed a $100 \%$ decrease in brood size at all the exposure hrs. Trichlorfon also significantly reduced the offspring count by 100,82 , and $100 \%$ at 24,48 , and $72 \mathrm{hrs}$ of exposure respectively. Disulfoton induces offspring reduction by 71,55 , and $60 \%$ respectively at 24,48 , and $72 \mathrm{hrs}$ of exposure.

\subsection{Learning}

While evaluating learning behaviour from table 1, disulfoton showed attraction of treated worm towards solvent well i.e., nonfood source rather than food source within 4 minutes, showing the inability to differentiate into food and non-food source whereas, worms in all the control plate reached towards the food source within 2 minutes. Worms in the other two pesticide exposures showed no attraction towards any well, providing defective learning at a higher degree.

\subsection{Mortality}

A concentration-dependent reduction in the survival of worms was reported in the figure1(D) in all OP tested pesticides. A steep reduction was observed since the 24 hrs of exposure with $67 \%$, $56 \%$, and $78 \%$ mortality when compared from control in chlorpyrifos, disulfoton, and trichlorfon respectively. By the exposure period of $48 \mathrm{hrs}$, chlorpyrifos showed $88 \%$ reduction in the survival rate followed by $50 \%$ and $75 \%$ in disulfoton and trichlorfon with control. Chlorpyrifos induced $100 \%$ mortality by the completion of $72 \mathrm{hrs}$ while it was reported $57 \%$ in disulfoton and $71 \%$ in trichlorfon.

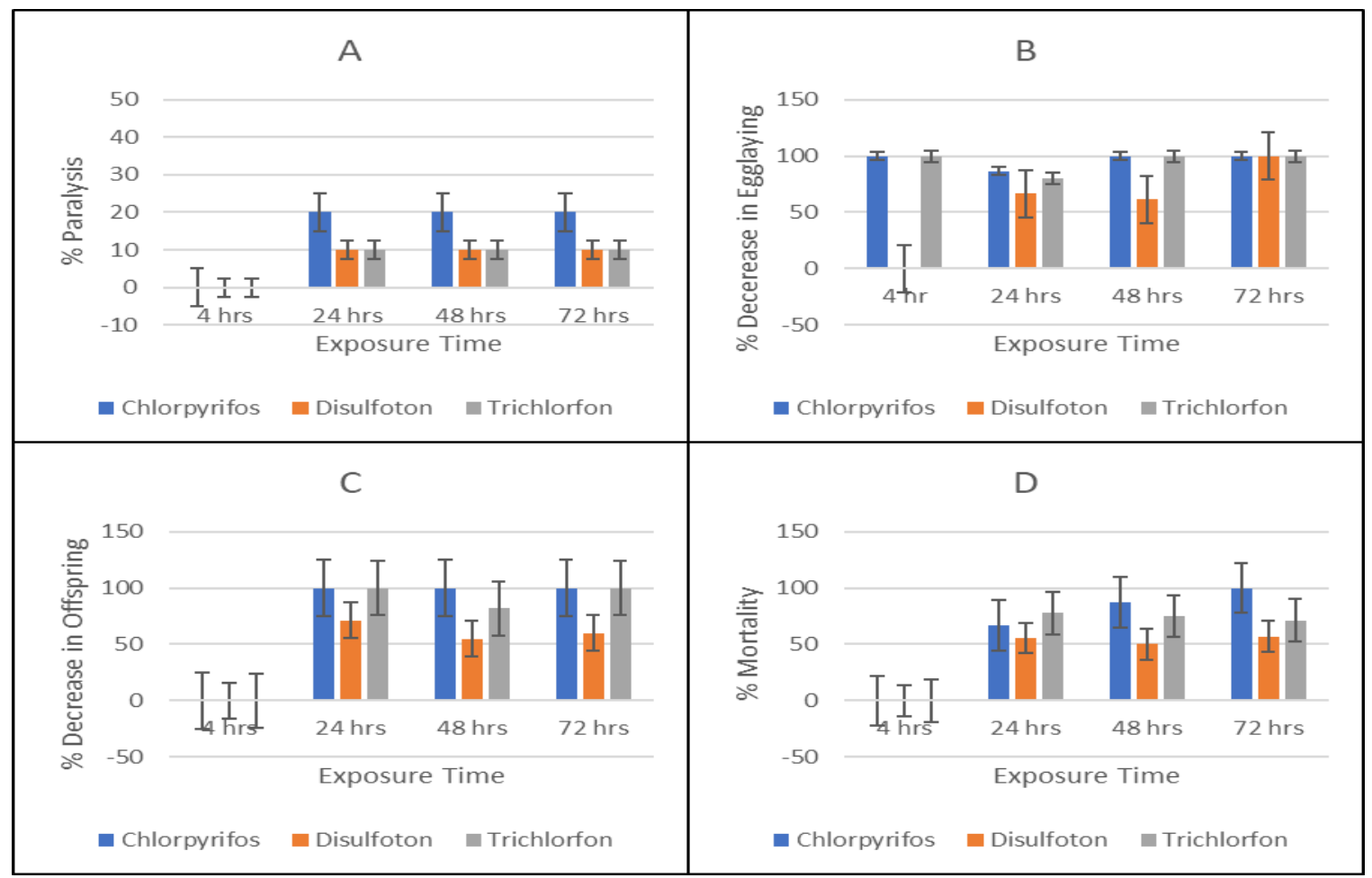

Figure 1 (A) Percent paralysis, (B) Percent decrease in Egg-laying of the worms, (C) Percent decrease in the Offspring count of the worms, and (D) Percent Mortality of the worms when treated against OPs.

Journal of Experimental Biology and Agricultural Sciences http://www.jebas.org 


\subsection{Body Length}

An adult worm takes around 3-4 days to develop from an egg. Exposing the first larval stage (L1) of the worm to pesticides produces a direct effect on its growth. As shown in Table 1, the body length of a healthy worm is $\sim 1 \mathrm{~mm}$. Chlorpyrifos rendered the worms to their first larval stage (L1) while disulfoton did not show any significant difference in the body length of the worms. Trichlorfon delayed the growth of the worms, rendering them to be in L4 stage rather than adulthood. The overall affectedness of the treated pesticides over all the quantified parameters is shown in figure 2 .

Table 1: Effect of OPs on learning and growth of C. elegans

\begin{tabular}{|ccccc|}
\hline Parameters & Control & Chlorpyrifos & Disulfoton & Trichlorfon \\
\hline Learning Outcome & $\begin{array}{c}\text { Reached to the food source } \\
\text { within 2 minutes }\end{array}$ & $\begin{array}{c}\text { No movement towards } \\
\text { the food }\end{array}$ & $\begin{array}{c}\text { 4 minutes to dwell in } \\
\text { non-food source }\end{array}$ & $\begin{array}{c}\text { No movement } \\
\text { towards the food }\end{array}$ \\
\hline Body Length & $\sim 1 \mathrm{~mm}$ & Ceased to L1 & $1 \mathrm{~mm}$ & $0.9 \mathrm{~mm}$ \\
\hline
\end{tabular}

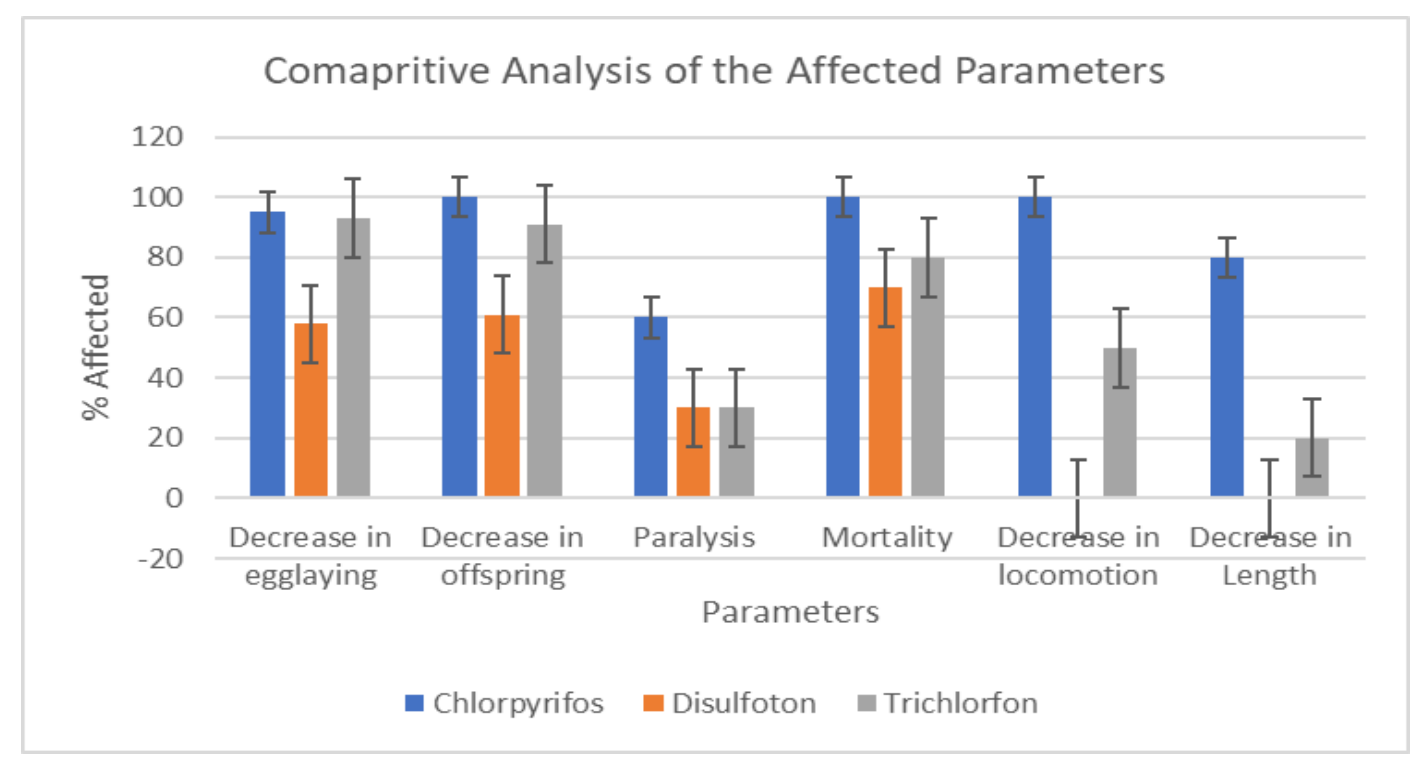

Figure 2 Overall comparative analysis of quantified parameters due to OPs.

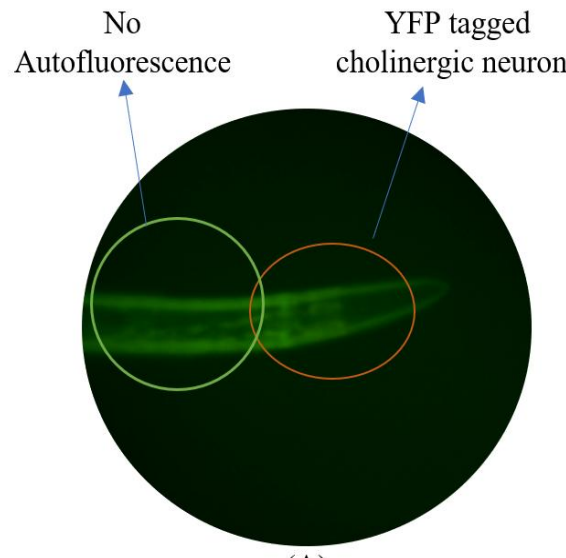

(A)

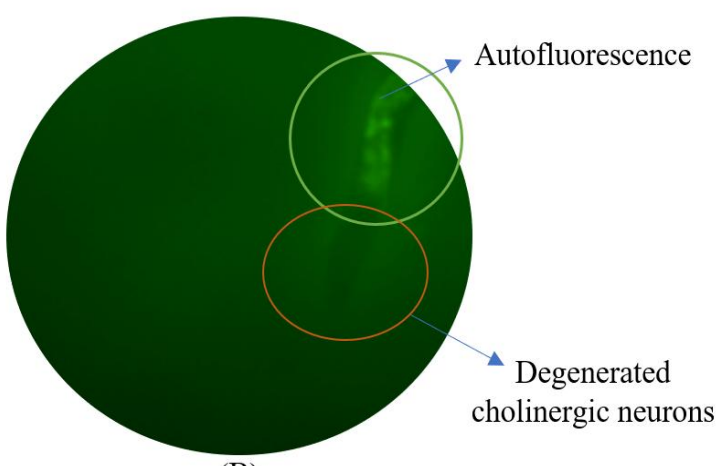

(B)

Figure 3 (A) OH11124 control worm displaying YFP tagged cholinergic neurons on the body walls with no autofluorescence in the gut at $72 \mathrm{hrs}$; (B) OH11124 pesticide treated worm displaying no GFP tagged cholinergic neurons on the body wall with

Journal of Experimental Biology and Agricultural Sciences http://www.jebas.org 


\subsection{Neurodegeneration and Autofluorescence}

Effect of OPs on Neurodegeneration was tested using Trichlorfon with transgenic strains OH11124 (otIs388 [eat4(fosmid)::SL2::YFP::H2B + (pBX) pha-1(+)] III) and OH13083 (otIs576 [unc-17(fosmid)::GFP + lin-44::YFP]) to check degeneration of cholinergic (Figure 3) and glutamatergic neurons (Figure 4). Treated worms from transgenic strain $\mathrm{OH} 11124$ showed a decrease by $57.96 \%$ in its YFP intensity from control whereas OH13083 incurred a 30.52\% decrease in its GFP intensity from its control (Figure 5). The survived worms at $72 \mathrm{hrs}$ of trichlorfon exposure not only decreased the GFP intensity of the tagged neurons but also induced pronounced autofluorescence which is a marker for aging.

\section{Discussion}

The current study evaluated the $C$. elegans based approach for toxicological research as excessive and inefficient pesticides have become a global issue for the last few decades. Results of this study provide evidence of the toxic effects of OPs in C. elegans as measured in terms of locomotion, paralysis, egg-laying, reproduction, learning, mortality, growth, neurodegeneration, and autofluorescence. Alterations in all the above-mentioned endpoints signify neurophysiological and behavioural perturbations as a result of organophosphate toxicity.

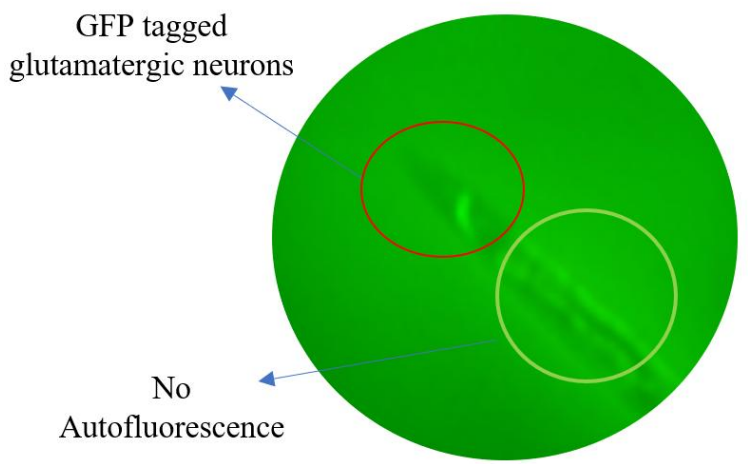

(A)

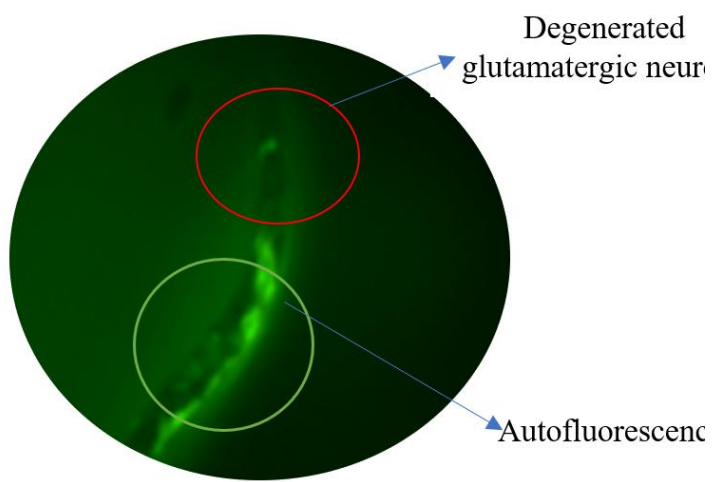

(B)

Figure 4 (A) OH13083 control worm displaying GFP tagged glutamatergic neurons with no intestinal autofluorescence at 72 hrs; (B) $\mathrm{OH} 13083$ treated worm displaying reduction in GFP intensity of glutamatergic neurons with intestinal autofluorescence at $72 \mathrm{hrs}$. Scale Bar $\sim 300 \mu \mathrm{m}$.

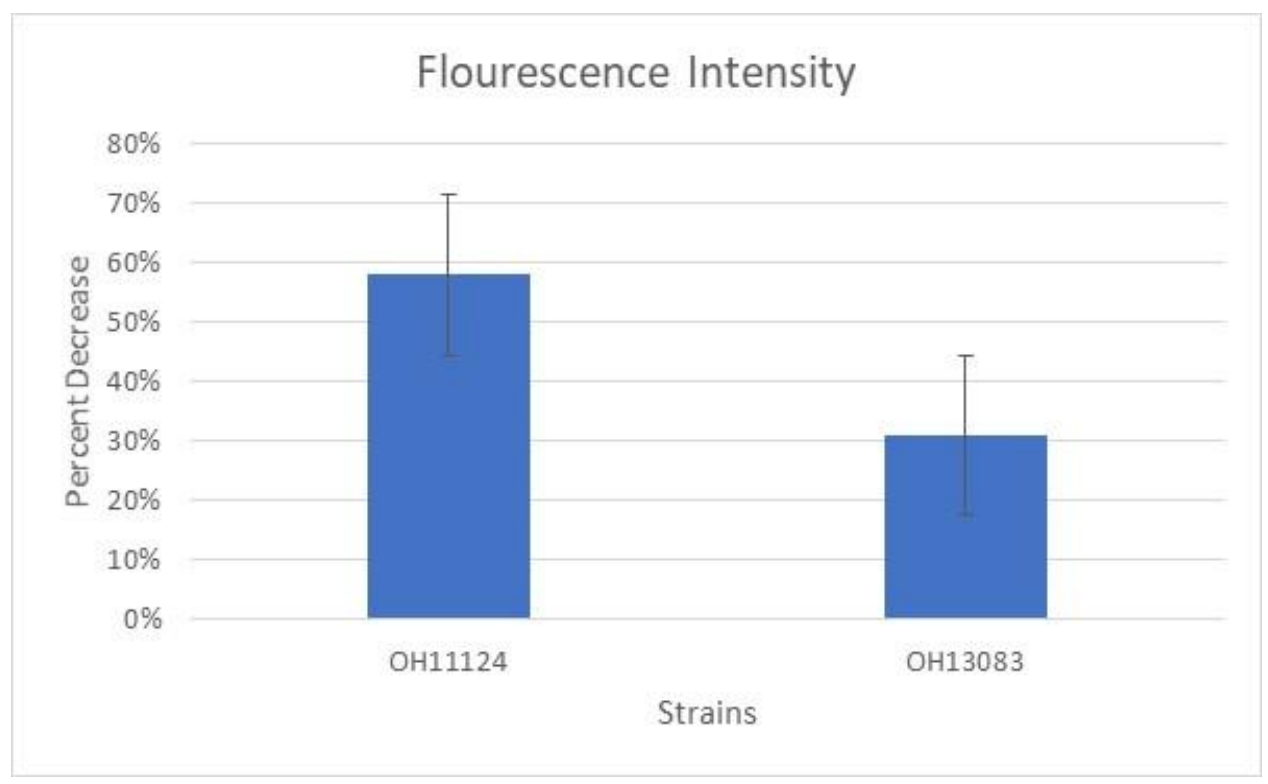

Figure 5 Data represent percent decrease in mean fluorescence intensity of Transgenic strains OH11124 and OH13083.

Journal of Experimental Biology and Agricultural Sciences http://www.jebas.org 
Locomotion results as an acetylcholine-associated behaviour, including the highest number of cholinergic neurons (Rand, 2007; Mille et al., 2021). From figure 2, it can be concluded that chlorpyrifos affected the locomotory behaviour which was followed by trichlorfon. Paralysis, a function of the cholinergic defect was clearly stated by Leelaja \& Rajini (2013). Similarly, levamisole and Aldicarb have also been reported to induce paralysis in C. elegans (Nonet et al., 1998). This study also showed that chlorpyrifos caused significant paralysis to the worms than trichlorfon and disulfoton. The above data support that OPs induced paralysis and decreased locomotion are sensitive as well as essential endpoints in toxicity testing which coincides with the study from Meyer \& Williams, 2014. Egg-laying behaviour in $C$. elegans has been proved as an excellent behavioural endpoint, not just because it has been well characterized, but also because it can be quantified effectively. Results incorporated in figure 2 revealed that chlorpyrifos and trichlorfon were found highly effective against the egg-laying behaviour of the worms throughout the different exposure periods. There are multiple mechanisms involving heterogenous neurotransmitters and ACh that affect egglaying (Bany et al., 2003; Bradford et al., 2020). In the findings of the current study, OPs exposure to the worms induced a significant decrease in the rate of egg-laying, a phenomenon concurrent with reports marking egg-laying defects that trigger neurodegeneration pathways (Caldwell et al., 2020).

Exposure of worms to OPs has also been reported in a study conducted by Leelaja \& Rajini (2013), whereas paralysis was induced in the worms along with the decrease in brood size due to exposure to sublethal concentrations of the organophosphorus insecticide, monocrotophos. This study showed that the chlorpyrifos and trichlorfon not only decreased the egg count of the worms but also ceased larval stages of development. Similar brood size reduction results were reported in the study by various previous researchers (Bradford et al., 2020; Wen et al., 2020).

C. elegans is known to adapt mechanosensory, olfactory, gustatory, and thermosensory behaviours through associative and non-associative learning, as a result of sensory inputs altogether (Walker et al., 2017). In the present study, the inability of worms to differentiate between the food source and the non-food source was analyzed. The control worms could quickly trace their food source within 2 minutes when kept on a test plate, but the OPs exposed worms could not find a way to their food i.e., $E$. coli OP50 filled well. As worms did not show any locomotory behaviour when kept equidistant from test wells, and it was significant that chlorpyrifos and trichlorfon caused a defect in their olfaction verifying defective learning and memory. The pesticide driven toxicity was also seen in disulfoton where worms did not reach their food and kept wandering in the plate away from food. Similar altered learning and memory studies were seen in a study stimulated by MCP and glucose by Salim \& Rajini, 2014. Further, Peris-Sampedro et al. (2015) also compiled that repeated exposure to chlorpyrifos led to memory impairment in the mice model.

Results from the growth endpoint validate that chlorpyrifos not only hindered the growth but also ceasing the development of the worms in the L1 stage. Trichlorfon also delayed the development of the worm which restricted them to attain adulthood in the given time, while worms from disulfoton did not affect the growth and development of the worms. This suggests that chlorpyrifos and trichlorfon severely affect the growth and development of the worms triggering neurodegeneration (Alexander et al., 2014). A study from Zhuang et al. (2014) also noted a significant reduction in the adult stage when the L1-larvae of C. elegans were exposed to ractopamine and clenbuterol (Zhuang et al., 2014). Similarly, findings explored body length as a parameter to evaluate body length post chemical exposure and noted the coinciding results (Meyer \& Williams, 2014; Bradford et al., 2020).

Mortality is a customarily assessed endpoint in toxicant studies. Results of the study revealed that Chlorpyrifos had a gradual reduction in the survival of worms whereas trichlorfon incurred overall mortality of $80 \%$ worms while disulfoton on the other hand showed $70 \%$ mortality. This increased mortality in the worms indicates the acute toxicity associated with the OPs. These findings recapitulated the results attained by Bradford et al. (2020) with the neonicotinoids exposed worms.

In C. elegans, lipofuscin deposits in lysosomes accumulate over time in the intestine and uterus producing autofluorescence. This phenomenon tends to accelerate with oxidative stress, heat fluctuations, and aging (Zhuang et al., 2014; Teuscher \& Ewald, 2018). Although chlorpyrifos was the most affecting OP, for neurodegenerative and autofluorescence studies, trichlorfon was taken forward because of $100 \%$ mortality in chlorpyrifos at $72 \mathrm{hrs}$ of exposure. Comparing OP treated worms over control; it was found that trichlorfon induced early autofluorescence which was not seen in control worms at that time. This signifies that OP can trigger the aging process parallel to neurodegeneration (Caldwell et al., 2020). Similarly, Zhuang et al. (2014) validated intestinal autofluorescence along with the defect in locomotion and brood size endpoints when exposed to chronic ractopamine and clenbuterol treatment. C. elegans has immersed as a pertinent model organism for studying neurodegenerative diseases like Alzheimer's and Parkinson's by focusing on their causes, progression, and therapeutics (Ju et al., 2010; Ruszkiewicz et al., 2018; Caldwell et al., 2020). Moreover, these associations of the behaviours of the nematode has been feebly connected to cholinergic and glutamatergic neurons (Jadhav \& Rajini, 2009b; Chen et al., 2015; Silveira et al., 2018). 
As every behaviour is associated with complex neural networks, the literature suggests that the most basic affected behaviours are associated with cholinergic and glutamatergic neurons (Jadhav \& Rajini, 2009a; Abdelhack, 2016; Caldwell et al., 2020). Therefore to correlate and evaluated the association of the neurotoxic effect of these OPs, transgenic strains OH11124 (otIs388 [eat4(fosmid)::SL2::YFP::H2B + (pBX) pha-1(+)] III)and OH13083 (otIs576 [unc-17(fosmid)::GFP + lin-44::YFP]) were chosen for glutamatergic and cholinergic neurons with tagged GFP. The eat- 4 is a mammalian homolog gene associated with glutamate processing glutamatergic neurotransmission (Brockie, 2006; Chen et al., 2013) It is expressed in the body wall of the worm, where its mutant strains verify defects in glutamatergic neurotransmission (Chen et al., 2013; Wen et al., 2020). The unc-17 is a cholinergic promoter that regulates a complex gene locus involved in acetylcholine synthesis and vesicle-loading of the cholinergic neurotransmission (Rand, 2007; Pereira et al., 2015). Current study results of neurogenerative along with autofluorescence test confirm that the prevailing OP i.e., trichlorfon not only causes degeneration of associated neurons but also induces autofluorescence as a marker of aging, correlating the etiology of neurodegenerative disease like Alzheimer's.

\section{Conclusion}

Environmental toxicity has been extensively studied to assess its adverse effects on behavioural changes using C. elegans. The present research focuses on evaluating the effects of toxicants at lower concentrations of organophosphates over a variable range of exposure periods. It was found that chlorpyrifos was the most affecting OP while this was followed by trichlorfon when tested on varied parameters. Most affected and reliable parameters were found to egg-laying, mortality, and neurodegeneration. Overall behavioural and developmental parameters infer to have a direct co-relationship on neurons that verifies the dysfunction and degeneration of the target site. Therefore, changes in these endpoints can be linked to their associated neurons and this linkage underlines the necessity of further research to understand the mechanism by which OPs cause neurophysiological alteration at the cellular and molecular levels. Further by associating this piece of information with humans, we can check over the probable inducers of neurodegenerative diseases as the basic framework of neurons is fundamentally conserved in the two species. The concentrations of OPs used in the research (under the permissible limits for many available commercial products) with a vast range of exposure duration make this data more valuable and pave the way for a detailed study and identification of the toxic effects of OPs in humans.

\section{Acknowledgments}

C. elegans $\mathrm{N} 2$ strain used in this study was kindly provided by the Caenorhabditis Genetics Center (CGC), MN, USA. This work was funded by DST- SERB [EEQ/2016/000590].

\section{Conflict of interest}

The authors declare that they have no conflicts of interest.

\section{Reference}

Abdelhack M (2016) Dopaminergic neurons modulate locomotion in Caenorhabditis elegans. bioRxiv 056192.

Alexander AG, Marfil V, Li C (2014) Use of Caenorhabditis elegans as a model to study Alzheimer's disease and other neurodegenerative diseases. Frontiers in genetics 5:1-21. https://doi.org/10.3389/fgene.2014.00279.

Bany IA, Dong MQ, Koelle MR (2003) Genetic and cellular basis for acetylcholine inhibition of Caenorhabditis elegans egg-laying behavior. Journal of Neuroscience 23:8060-8069. https://doi.org/10.1523/jneurosci.23-22-08060.2003.

Bradford BR, Whidden E, Gervasio ED, Checchi PM, Raley-Susman KM (2020) Neonicotinoid-containing insecticide disruption of growth, locomotion, and fertility in Caenorhabditis elegans. PLoS ONE 15:1-21. https://doi.org/10.1371/journal.pone.0238637.

Brockie P (2006) Ionotropic glutamate receptors: genetics, behavior and electrophysiology. WormBook 1-16. https://doi.org/10.1895/wormbook.1.61.1.

Caldwell KA, Willicott CW, Caldwell GA (2020) Modeling neurodegeneration in Caenorhabditis elegans. Disease Models \& Mechanisms 13. https://doi.org/10.1242/dmm.046110.

Chen P, Martinez-Finley EJ, Bornhorst J, Chakraborty S, Aschner M (2013) Metal-induced neurodegeneration in C . elegans. Frontiers in Aging Neuroscience 5:1-11. https://doi.org/10.3389/fnagi.2013.00018.

Chen X, Barclay JW, Burgoyne RD, Morgan A (2015) therapeutic compounds for ageing - associated neurodegenerative diseases. Chemistry Central Journal 1-20. https://doi.org/10.1186/s13065015-0143-y.

Cole RD, Anderson GL, Williams PL (2004) The nematode Caenorhabditis elegans as a model of organophosphate-induced mammalian neurotoxicity. Toxicology and Applied Pharmacology 194:248-256. https://doi.org/10.1016/j.taap.2003.09.013.

Francis PT, Palmer AM, Snape M, Wilcock GK (1999) The cholinergic hypothesis of Alzheimer's disease: a review of progress. Neurol Neurosurg Psychiatry 66:137-147.

Hargreaves AJ (2012) Neurodegenerations induced by organophosphorous compounds. Advances in Experimental 
Medicine and Biology 724:189-204. https://doi.org/10.1007/9781-4614-0653-2_15.

Harlow PH, Perry SJ, Widdison S, Daniels S, Bondo E, Lamberth C, Currie RA, Flemming AJ (2016) The nematode Caenorhabditis elegans as a tool to predict chemical activity on mammalian development and identify mechanisms influencing toxicological outcome. Scientific reports 6:1-13. https://doi.org/10.1038/ srep22965.

Jadhav KB, Rajini PS (2009a) Neurophysiological alterations in Caenorhabditis elegans exposed to dichlorvos, an organophosphorus insecticide. Pesticide Biochemistry and Physiology 94:79-85. https://doi.org/10.1016/j.pestbp.2009.03.004.

Jadhav KB, Rajini PS (2009b) Evaluation of sublethal effects of dichlorvos upon Caenorhabditis elegans based on a set of end points of toxicity. Pesticide Biochemistry and Physiology 23:9-17. https://doi.org/10.1002/jbt.20258.

Jain S, van Kesteren RE, Heutink P (2012) High Content Screening in Neurodegenerative Diseases. Journal of Visualized Experiments 1-9. https://doi.org/10.3791/3452.

John H, Richter A, Siegert M, Eyer F, Thiermann H (2021) Evidence of exposure to organophosphorus toxicants by detection of the propionylated butyrylcholinesterase-derived nonapeptideadduct as a novel biomarker. Forensic Science International 323:110818. https://doi.org/10.1016/j.forsciint.2021.110818.

Ju JJ, Ruan QL, Li YH, Liu R, Yin LH, Pu YP (2010) Neurotoxicity Evaluation of Chlorpyrifos Exposure with Caenorhabditis elegans. In 2010 4th International Conference on Bioinformatics and Biomedical Engineering (Pp 1-4) IEEE.

Leelaja BC, Rajini PS (2013) Biochemical and physiological responses in Caenorhabditis elegans exposed to sublethal concentrations of the organophosphorus insecticide, monocrotophos. Ecotoxicology and environmental safety 94:8-13. https://doi.org/10.1016/j.ecoenv.2013.04.015.

Lewis JA, Gehman EA, Baer CE, Jackson DA (2013) Alterations in gene expression in Caenorhabditis elegans associated with organophosphate pesticide intoxication and recovery. BMC Genomics 14(1):1:17.

Meyer-Baron M, Knapp G, Schäper M, van Thriel C (2015) Metaanalysis on occupational exposure to pesticides - Neurobehaviora impact and dose-response relationships. Environmental Research 136:234-245. https://doi.org/10.1016/j.envres.2014.09.030.

Meyer D, Williams PL (2014) Toxicity Testing of Neurotoxic Pesticides in Caenorhabditis elegans. Journal of Toxicology and
Environmental Health, Part B

https://doi.org/10.1080/10937404.2014.933722.

Mille T, Quilgars C, Cazalets JR, Bertrand SS (2021) Acetylcholine and spinal locomotor networks: The insider. Physiological Reports 9:1-10. https://doi.org/10.14814/phy2.14736.

Monici M (2005) Cell and tissue autofluorescence research and diagnostic applications. Biotechnology Annual Review 11:227256.https://doi.org/10.1016/S1387-2656(05)11007-2.

Muir JL (1997) Alzheimer , s Disease. Pharmacology Biochemistry and Behavior 56:687-696.

Mukherjee S, Gupta RD (2020) Organophosphorus Nerve Agents: Types, Toxicity, and Treatments. Journal of Toxicology. https://doi.org/10.1155/2020/3007984.

Muñoz-Quezada MT, Lucero BA, Iglesias VP, Muñoz MP, Cornejo CA, Achu E, Baumert B, Hanchey A, Concha C, Brito AM, Villalobos M (2016) Chronic exposure to organophosphate (OP) pesticides and neuropsychological functioning in farm workers: a review. International Journal of Occupational and Environmental Health 22(1):68-79. https://doi.org/10.1080/10773525.2015.1123848.

Nonet ML, Saifee O, Zhao H, Rand JB, Wei L (1998) Synaptic transmission deficits in Caenorhabditis elegans synaptobrevin mutants. Journal of Neuroscience 18:70-80. https://doi.org/10.1523/jneurosci.18-01-00070.1998.

Pereira L, Kratsios P, Serrano-Saiz E, Sheftel H, Mayo AE, Hall DH, White JG, LeBoeuf B, Garcia LR, Alon U, Hobert O (2015) A cellular and regulatory map of the cholinergic nervous system of $\mathrm{C}$. elegans. Elife 4: e12432 1-46. https://doi.org/10.7554/eLife.12432.

Peris-Sampedro F, Basaure P, Reverte I, Cabré M, Domingo JL, Colomina MT (2015) Chronic exposure to chlorpyrifos triggered body weight increase and memory impairment depending on human apoE polymorphisms in a targeted replacement mouse model. Physiology and Behavior 144:37-45. https://doi.org/10.1016/j.physbeh.2015.03.006.

Pincus Z, Mazer TC, Slack FJ (2016) Autofluorescence as a measure of senescence in C. elegans: Look to red, not blue or green. Aging 8:889-898. https://doi.org/10.18632/aging.100936.

Queirós L, Pereira JL, Gonçalves FJ, Pacheco M, Aschner M, Pereira P (2019) Caenorhabditis elegans as a tool for Environmental Risk Assessment - emerging and promising applications for a "nobelized worm." Physiology \& Behavior 176:139-148.

https://doi.org/10.1080/10408444.2019.1626801.Caenorhabditis. 
Rajini PS, Melstrom P, Williams PL (2008) A comparative study on the relationship between various toxicological endpoints in Caenorhabditis elegans exposed to organophosphorus insecticides. Journal of Toxicology and Environmental Health - Part A 71:1043-1050. https://doi.org/10.1080/15287390801989002.

Rand J (2007) Acetylcholine. WormBook 1-21. https://doi.org/ 10.1895/wormbook.1.131.1.

Reynoso EC, Torres E, Bettazzi F, Palchetti I (2019) Trends and perspectives in immunosensors for determination of currently-used pesticides: The case of glyphosate, organophosphates, and neonicotinoids. Biosensors 9:. https://doi.org/10.3390/bios9010020.

Roh JY, Choi J (2011) Cyp35a2 gene expression is involved in toxicity of fenitrothion in the soil nematode Caenorhabditis elegans. Chemosphere 84:1356-1361. https://doi.org/10.1016/ j.chemosphere.2011.05.010.

Roldan-Tapia L, Nieto-Escamez FA, del Aguila EM, Laynez F, Parron T, Sanchez-Santed F (2006) Neuropsychological sequelae from acute poisoning and long-term exposure to carbamate and organophosphate pesticides. Neurotoxicology and Teratology 28(6):94-703. https://doi.org/10.1016/j.ntt.2006.07.004.

Ruszkiewicz JA, Pinkas A, Miah MR, Weitz RL, Lawes MJ, Akinyemi AJ, Ijomone OM, Aschner M (2018) C . elegans as a model in developmental neurotoxicology. Toxicology and Applied Pharmacology 354:126-135. https://doi.org/10.1016/j.taap. 2018.03.016.

Salim C, Rajini PS (2014) Glucose feeding during development aggravates the toxicity of the organophosphorus insecticide Monocrotophos in the nematode, Caenorhabditis elegans. Physiology and Behavior 131:142-148. https://doi.org/10.1016/ j.physbeh.2014.04.022.

Sánchez-Santed F, Colomina MT, Herrero Hernández E (2016) Organophosphate pesticide exposure and neurodegeneration. Cortex 74:417-426. https://doi.org/10.1016/j.cortex.2015.10.003.
Silveira da TL, Zamberlan DC, Arantes LP, Machado ML, da Silva TC, de Freitas Câmara D, Santamaría A, Aschner M, Soares FA (2018) Quinolinic Acid and glutamatergic neurodegeneration in Caenorhabditis elegans. Neurotoxicology 67:94-101. https://doi.org/10.1016/j.neuro.2018.04.015.

Stiernagle T (2006) Maintenance of C. elegans. WormBook 1-11. https://doi.org/10.1895/wormbook.1.101.1.

Tejeda L, Olivero-verbel J, Tejeda-benitez L, Olivero-verbel J (2016) Caenorhabditis elegans, a Biological Model for Research in Toxicology. Reviews of Environmental Contamination and Toxicology 237. https://doi.org/10.1007/978-3-319-23573-8.

Terry AV (2012) Functional consequences of repeated organophosphate exposure: Potential non-cholinergic mechanisms. Pharmacology and Therapeutics 134:355-365. https://doi.org/10.1016/j.pharmthera.2012.03.001.

Teuscher AC, Ewald CY (2018) Europe PMC Funders Group Overcoming Autofluorescence to Assess GFP Expression During Normal Physiology and Aging in Caenorhabditis elegans. Bioprotocol 8. https://doi.org/10.21769/BioProtoc.2940.Overcoming.

Walker DS, Chew YL, Schafer WR (2017) Oxford Handbooks Online Genetics of Behavior in C. elegans.

Wen X, Chen YH, Li R, Ge MH, Yin SW, Wu JJ, Huang JH, Liu H, Wang PZ, Gross E, Wu ZX (2020). Signal Decoding for Glutamate Modulating Egg Laying Oppositely in Caenorhabditis elegans under Varied Environmental Conditions. Iscience, 23(10), 101588. https://doi.org/10.1016/ j.isci.2020.101588.

Zhuang Z, Zhao Y, Wu Q, Li M, Liu H, Sun L, Gao W, Wang D (2014) Adverse effects from clenbuterol and ractopamine on nematode Caenorhabditis elegans and the underlying mechanism. PLoS ONE 9:1-11. https://doi.org/10.1371/journal.pone.0085482.

Journal of Experimental Biology and Agricultural Sciences http://www.jebas.org 